\title{
Venous Thromboembolic Diseases: Diagnosis, Management and Thrombophilia Testing: Observations on NICE Guideline [NG158]
}

\author{
${ }^{1}$ Department of Medicine, Thrombosis and Atherosclerosis Research \\ Institute, McMaster University, Hamilton, Ontario, Canada \\ ${ }^{2}$ Department of Obstetrics and Gynecology, I.M. Sechenov First \\ Moscow State Medical University, Moscow, Russia \\ ${ }^{3}$ Center for Thrombosis and Hemostasis, University Medical Center \\ Mainz, Mainz, Germany \\ ${ }^{4}$ Department of Cardiology, Democritus University of Thrace, Thrace, \\ Greece \\ ${ }^{5}$ Institute of Hematology, Union Hospital, Tongji Medical College, \\ Huazhong University of Science and Technology, Wuhan, China
}

Sam Schulman ${ }^{1,2}$ Stavros Konstantinides ${ }^{3,4}$ Yu Hu ${ }^{5}$ Liang V. Tang ${ }^{5}$
Thromb Haemost 2020;120:1143-1146.

The new edition of the National Institute for Health and Care Excellence (NICE) guideline (NG158) regarding venous thromboembolic diseases (VTDs) was published on March 26, 2020 (https://www.nice.org.uk/guidance/ng158). Given the common occurrence and health care burden of venous thromboembolism (VTE) in clinical practice, as well as the heterogeneity in its presentations, risk factors, and outcomes, ${ }^{1-3}$ the NICE guidance provides welcomed evidence-based recommendations, based on systematic reviews, evidence appraisal, and cost effectiveness. This approach is different from some other guidance, which may be more eminence-based rather than having a formally appraised evidence-base.

Compared with the previous edition, there are some changes and new recommendations on diagnosis and management of VTD in adults ( - Fig. $\mathbf{1}$ ).

The first main change we would like to discuss here is pulmonary embolism rule-out criteria (the PERC rule). Pulmonary embolism (PE) is a potentially fatal disease with various and unspecific presentations, ranging from vague chest discomfort with normal vital signs to sudden death. As a result, Ddimer test and interim anticoagulation treatment are often applied even when the clinician estimates the probability of PE as very low. ${ }^{1}$ The PERC rule is derived to provide safe exclusion criteria for suspected PE with a likelihood lower than $15 \%$ based on a gestalt impression. ${ }^{4}$ It is an eight-variable decision rule and the PERC negative, which is obtained when the answer to all the eight questions is "no," indicates no further investigation. In a multicenter, prospective, observational study in emergency departments, patients with suspected PE, a low

Address for correspondence Sam Schulman, MD, PhD, Thrombosis Service, HHS-General Hospital, Hamilton, ON L8L 2X2, Canada (e-mail: schulms@mcmaster.ca).

implicit clinical probability and PERC negative assessment had a PE prevalence of $1.2 \%$ (95\% confidence interval, 0.4-2.9). A total of $4.7 \%$ patients developed PE within a 3-month followup, and the proportion of patients meeting the rule was $32 \%{ }^{5}$ So far this rule is mainly used in emergency departments. Having to meet all the eight criteria may also limit the usefulness of this rule, and whether modified PERC would have better performance absolutely requires further validation before put into clinical application.

Recently, a simplified diagnostic pathway-the YEARS algorithm-has been proposed and found useful in suspected PE. ${ }^{6}$ It includes three items (clinical signs of deep vein thrombosis [DVT], hemoptysis, considering PE as the most likely diagnosis) and D-dimer level. When the D-dimer is $<500 \mu \mathrm{g} / \mathrm{L}$ or $<1,000 \mu \mathrm{g} / \mathrm{L}$ in the absence of any item, PE is excluded. Otherwise, examination with computed tomography of the pulmonary arteries (CTPA) is required for further diagnosis. In a prospective, multicenter, cohort study of 3,616 patients with suspected PE, 48\% were managed without CTPA, compared with $34 \%$ when Wells rule and conventional D-dimer threshold were used. ${ }^{7}$ The false-negative rate was less than $1 \%$. Further validation is still needed before putting the YEARS diagnostic algorithm into clinical practice.

The NICE guideline proposes outpatient treatment for suspected or confirmed low-risk PE, using a validated risk stratification tool. Indeed, outpatient management is common in U.K. health care setting (as with many other countries). To facilitate the decision process, the 2019 European Society of Cardiology guidelines for the

(c) 2020 Georg Thieme Verlag KG Stuttgart · New York
DOI https://doi.org/ $10.1055 / \mathrm{s}-0040-1712913$. ISSN 0340-6245. 


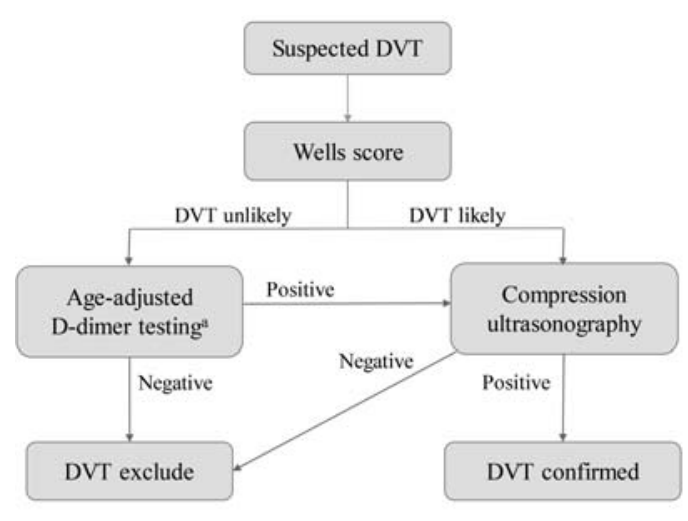

A

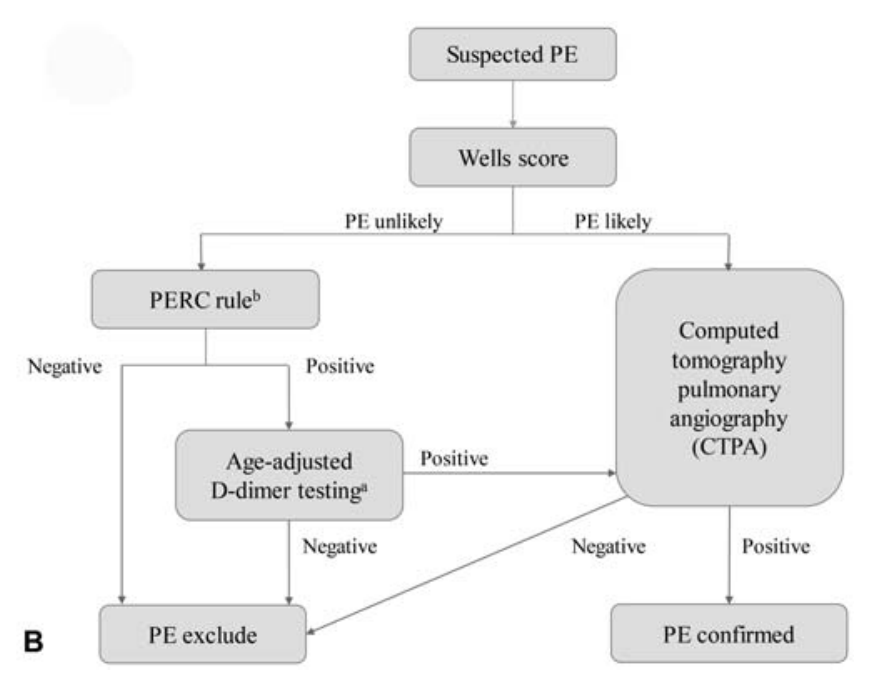

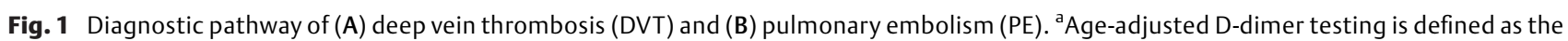
patient's age multiplied by $10 \mu \mathrm{g} / \mathrm{L}$ for patients aged over 50 , and $500 \mu \mathrm{g} / \mathrm{L}$ for younger patients, respectively. The optimal thresholds may vary for different D-dimer assays.

management of PE defined three sets of criteria for considering early discharge and home treatment. ${ }^{8}$ These include (1) a low risk of early PE-related serious complications; (2) absence of serious comorbidity or aggravating conditions; and (3) proper outpatient care, taking into account the patient's anticipated compliance, family and social environment, and the local infrastructure. ${ }^{8}$ The Pulmonary Embolism Severity Index integrates clinical parameters of PE severity and comorbidity, and may be used as a tool for selecting candidates for home treatment, if combined with additional feasibility criteria. ${ }^{9}$ As an alternative, the Hestia exclusion criteria represent a checklist of clinical parameters that can be obtained at the bedside. ${ }^{10,11}$ Beyond clinical findings alone, a recent meta-analysis ${ }^{12}$ as well as a prospective management trial ${ }^{13}$ suggest that it is also wise to exclude right ventricular dysfunction and free floating right heart thrombi before discharge from hospital.

Before starting treatment of VTD, the NICE guideline recommends that hepatic function is included among the blood tests. A very small proportion of patients would be excluded from using oral anticoagulants based on poor liver function. Hepatic function tests should be limited to the few with suspected severe liver cirrhosis, where this is meaningful to assess contraindication to nonvitamin K antagonist oral anticoagulants (NOACs), and then ascites and encephalopathy should also be evaluated to calculate Child-Pugh score and exclude those with grade $\mathrm{C}$.

For the initial anticoagulation, apixaban or rivaroxaban are favored, which is indeed the clinical routine in many countries. This is followed by the statement that if "neither apixaban nor rivaroxaban is suitable offer: low molecular weight heparin (LMWH) for at least 5 days followed by dabigatran or edoxaban." It is understandable that LMWH may be preferable to apixaban or rivaroxaban initially in case of large PE, where thrombolysis might become indicated, or for very large DVT for similar reason, or if there is acute kidney injury that is expected to improve quickly, but a moot point beyond the clinical trial or the prescribing label, is why would dabigatran or edoxaban be better than any of the other two NOACs, after the 5 days of LMWH?

For patients with extreme body weight, NICE does not exclude NOACs but suggests "regular monitoring of therapeutic levels for people with confirmed proximal DVT or PE who weigh less than $50 \mathrm{~kg}$ or more than $120 \mathrm{~kg}$, to ensure effective anticoagulation." It is probably sufficient to confirm once, after steady state has been reached, that the level is well within the range seen in population pharmacokinetic studies. The NICE guideline is up to date with suggestion for NOACs in case of active cancer. For patients with severe renal failure (calculated creatinine clearance $<15 \mathrm{~mL} / \mathrm{min}$ ), LMWH is suggested as an option but without any dosing recommendations, referring the reader to local protocols or specialist advice. It is noteworthy that NICE has previously published guidelines specific for each of the approved NOACs as Technology Appraisals.

The recommended treatment duration is in line with other guidelines, 3 months for provoked VTE and up to 6 months if there is, for example, extensive thromboembolism. There is little specific guidance regarding the duration for other groups of patients, more than to take into account risks for bleeding, recurrence, adherence, patient values, and preferences. Regarding bleeding, NICE suggests "stopping anticoagulation if the HAS-BLED score is 4 or more and cannot be modified." The HAS-BLED score was originally derived and proposed for bleeding risk assessment in patients with atrial fibrillation (AF), ${ }^{14}$ and while some studies have tested the value of the HAS-BLED score for bleeding risk prediction in VTE, ${ }^{15,16}$ this recommendation from NICE is noteworthy. Nonetheless, bleeding risk assessment tools are often misused by the ill-informed as an excuse to stop anticoagulation, and inappropriate cessation can lead to poor outcomes. Instead, the HAS-BLED score should be used to draw attention to modifiable bleeding risk factors (e.g., uncontrolled blood pressure, concomitant use of nonsteroidal anti-inflammatory drugs or antiplatelets in an anticoagulated patient, labile international normalized ratios, etc.), 
and to flag up the high-risk patients for early review and follow-up (e.g., 4 weeks rather than 4-6 months). Such a proactive strategy with appropriate use of dynamic risk assessment and follow-up was associated with lowered bleeding events and improved anticoagulation uptake, at least among patients with AF. ${ }^{17}$

There are overall many suggestions regarding practical management, for example, provision of written information on signs and symptoms to be aware of, direct contact details to health care professionals, and which products contain components of animal origin-heparins, lactose from cow's milk in rivaroxaban and apixaban. The latter is pertinent for patients with specific religious/ethical beliefs or food intolerance.

The NICE guidance specifies the clinical setting in which catheter-directed thrombolytic therapy should be considered for symptomatic iliofemoral DVT. In acute PE, primary reperfusion treatment, in most cases systemic thrombolysis, is the treatment of choice for patients with hemodynamic instability. In contrast, for most cases of acute PE without hemodynamic compromise, the risk-to-benefit ratio of thrombolysis appears unfavorable. ${ }^{18}$ Catheter-directed treatment, including ultrasound-enhanced, catheter-directed thrombolysis, is mentioned by NICE as an alternative reperfusion option, particularly for patients with bleeding risk or other contraindications to systemic (full-dose) thrombolysis. The studies conducted so far have shown efficacy of this treatment as judged by surrogate endpoints (in most cases, fast reduction of right ventricular dimensions on follow-up imaging). ${ }^{19-21}$ Catheter-directed treatment now needs to be tested in adequately sized randomized trials with clinical primary outcomes. Independently from individual reperfusion options, an amendment to NICE recommendations would be that setup of multidisciplinary PE response teams should be encouraged. Coordinated, personalized treatment of patients with intermediate-high- and high-risk $\mathrm{PE}^{8}$ addresses the needs of modern systems-based health care. ${ }^{22}$

Reinforcing and amending its past recommendations, the NICE guideline wisely advises, like most international scientific societies, against routine cancer investigations (beyond physical examination and basic laboratory testing), and also against routine thrombophilia testing of unselected patients with "unprovoked" DVT or PE. In this context, it is important to emphasize that "provoked" DVT or PE requires the presence of a recent (within 3 months) and transient strong clinical risk factor for thrombosis. NICE includes pregnancy, puerperium, and hormonal therapy (including contraception) in the list of strong provoking factors, an opinion not fully shared by other guidelines. ${ }^{8}$ To avoid any misinterpretation of the NICE's balanced recommendations on thrombophilia testing, it can also be explicitly mentioned that there is no indication for routine antiphospholipid antibody testing as a prerequisite for starting or continuing treatment with a direct oral anticoagulant after DVT or PE.

\section{Conflict of Interest}

S.S. reports grants from Boehringer-Ingelheim and Octapharma, personal fees from Boehringer-Ingelheim, Bayer,
Daiichi, Alnylam, Sanofi, Bristol-Myers Squibb, and Pfizer, outside the submitted work.

S.K. reports grants and personal fees from Bayer AG, grants and personal fees from Daiichi Sankyo, personal fees from Pfizer-Bristol-Mayers Squibb, grants and personal fees from BTG - Boston Scientific Group, grants from Boehringer-Ingelheim, personal fees from MSD, grants and personal fees from Actelion - Janssen, grants from Servier, outside the submitted work.

\section{References}

1 Ageno W, Haas S, Weitz JI, et al; GARFIELD-VTE investigators. Characteristics and management of patients with venous thromboembolism: the GARFIELD-VTE registry. Thromb Haemost 2019; 119(02):319-327

2 Cohen AT, Agnelli G, Buller HR, et al. Characteristics and outcomes in patients with venous thromboembolism taking concomitant anti-platelet agents and anticoagulants in the AMPLIFY trial. Thromb Haemost 2019;119(03):461-466

3 Kalayci A, Gibson CM, Chi G, et al. Asymptomatic deep vein thrombosis is associated with an Increased risk of death: insights from the APEX trial. Thromb Haemost 2018;118(12):2046-2052

4 Kline JA, Slattery D, O'Neil BJ, et al. Clinical features of patients with pulmonary embolism and a negative PERC rule result. Ann Emerg Med 2013;61(01):122-124

5 Penaloza A, Soulié C, Moumneh T, et al. Pulmonary embolism rule-out criteria (PERC) rule in European patients with low implicit clinical probability (PERCEPIC): a multicentre, prospective, observational study. Lancet Haematol 2017;4(12): e615-e621

6 van der Pol LM, van der Hulle T, Mairuhu ATA, Huisman MV, Klok FA. Combination of pulmonary embolism rule-out criteria and YEARS algorithm in a European cohort of patients with suspected pulmonary embolism. Thromb Haemost 2018;118(03): $547-552$

7 van der Hulle T, Cheung WY, Kooij S, et al; YEARS study group. Simplified diagnostic management of suspected pulmonary embolism (the YEARS study): a prospective, multicentre, cohort study. Lancet 2017;390(10091):289-297

8 Konstantinides SV, Meyer G, Becattini C, et al; ESC Scientific Document Group. 2019 ESC guidelines for the diagnosis and management of acute pulmonary embolism developed in collaboration with the European Respiratory Society (ERS). Eur Heart J 2020;41(04):543-603

9 Aujesky D, Roy PM, Verschuren F, et al. Outpatient versus inpatient treatment for patients with acute pulmonary embolism: an international, open-label, randomised, non-inferiority trial. Lancet 2011;378(9785):41-48

10 den Exter PL, Zondag W, Klok FA, et al. Efficacy and safety of outpatient treatment based on the Hestia clinical decision rule with or without NT-proBNP testing in patients with acute pulmonary embolism: a randomized clinical trial. Am J Respir Crit Care Med 2016;194(08):998-1006

11 Zondag W, Mos IC, Creemers-Schild D, et al; Hestia Study Investigators. Outpatient treatment in patients with acute pulmonary embolism: the Hestia study. J Thromb Haemost 2011;9(08): 1500-1507

12 Barco S, Mahmoudpour SH, Planquette B, Sanchez O, Konstantinides SV, Meyer G. Prognostic value of right ventricular dysfunction or elevated cardiac biomarkers in patients with low-risk pulmonary embolism: a systematic review and meta-analysis. Eur Heart J 2019;40(11):902-910

13 Barco S, Schmidtmann I, Ageno W, et al; HoT-PE Investigators. Early discharge and home treatment of patients with low-risk pulmonary embolism with the oral factor $\mathrm{Xa}$ inhibitor 
rivaroxaban: an international multicentre single-arm clinical trial. Eur Heart J 2020;41(04):509-518

14 Pisters R, Lane DA, Nieuwlaat R, de Vos CB, Crijns HJ, Lip GY. A novel user-friendly score (HAS-BLED) to assess 1-year risk of major bleeding in patients with atrial fibrillation: the Euro Heart Survey. Chest 2010;138(05):1093-1100

15 Brown JD, Goodin AJ, Lip GYH, Adams VR. Risk stratification for bleeding complications in patients with venous thromboembolism: application of the HAS-BLED bleeding score during the first 6 months of anticoagulant treatment. J Am Heart Assoc 2018;7 (06):e007901

16 Kooiman J, van Hagen N, Iglesias Del Sol A, et al. The HAS-BLED score identifies patients with acute venous thromboembolism at high risk of major bleeding complications during the first six months of anticoagulant treatment. PLoS One 2015;10(04):e0122520

17 Guo Y, Lane DA, Chen Y, Lip GYH. Regular bleeding risk assessment associated with reduction in bleeding outcomes: the mAFA II randomised trial. Am J Med 2020;S0002-9343(20) 30274-6
18 Meyer G, Vicaut E, Danays T, et al; PEITHO Investigators. Fibrinolysis for patients with intermediate-risk pulmonary embolism. $\mathrm{N}$ Engl J Med 2014;370(15):1402-1411

19 Tapson VF, Sterling K, Jones N, et al. A randomized trial of the optimum duration of acoustic pulse thrombolysis procedure in acute intermediate-risk pulmonary embolism: the OPTALYSE PE trial. JACC Cardiovasc Interv 2018;11(14):1401-1410

20 Piazza G, Hohlfelder B, Jaff MR, et al; SEATTLE II Investigators. A prospective, single-arm, multicenter trial of ultrasound-facilitated, catheter-directed, low-dose fibrinolysis for acute massive and submassive pulmonary embolism: the SEATTLE II study. JACC Cardiovasc Interv 2015;8(10):1382-1392

21 Kucher N, Boekstegers P, Müller OJ, et al. Randomized, controlled trial of ultrasound-assisted catheter-directed thrombolysis for acute intermediate-risk pulmonary embolism. Circulation 2014; 129(04):479-486

22 Dudzinski DM, Piazza G. Multidisciplinary pulmonary embolism response teams. Circulation 2016;133(01):98-103 